\title{
Franz Liszt e la «Dante Symphony»: frammenti e simboli per una nuova musica "umanitaria»
}

\author{
Alessandro Avallone \\ Università di Roma "La Sapienza" \\ ale.falaut@iol.it
}

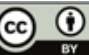

\section{Riassunto}

Negli anni trascorsi al servizio del Granduca di Weimar, caratterizzati dal desiderio di trovare un giaciglio sicuro per una profonda meditazione spirituale, Liszt ebbe modo di approfondire le proprie conoscenze sui materiali musicali non-pianistici, grazie alla compagine orchestrale messa a disposizione nel teatro di corte, e di maturare la propria concezione sansimonista dell'arte, intesa come straordinaria forza spirituale capace di unire l'umanità nel cammino del progresso sociale e civile. In questi anni si compie la concezione lisztiana della musica sinfonica "a programma", per cui la musica non può mai essere pura forma, piatta ed inespressiva, ma necessita sempre di un contenuto ideale forte, che solo la poesia può conferirle; l'ausilio del programma dantesco, cui Liszt si ispirò per la sua Dante Sympho$n y$, composta a Weimar nel 1856 , non va però inteso come omaggio al poema, bensì come lucida consapevolezza della sua modernità da parte del compositore: è la natura frammentaria e simbolica che viene còlta, escludendo significativamente l'ultima cantica, e in virtù del valore etico che la musica deve possedere, Liszt compie una selezione tra quelle immagini poetiche che egli ritiene più idonee ad essere rivestite del mezzo espressivo sinfonico.

Parole chiave: Liszt, musica a programma, sansimonismo, avvenirismo, poema sinfonico.

\begin{abstract}
:
Throughout his years at the service of the Grand Duke of Weimar, characterized by the desire to find a safe haven in which to quench his constant desire to be in deep spiritual meditation, Liszt was able to deepen his awareness of his own musical and non-pianistic material. Thanks to the orchestra available at the court theatre, he was able to mature his own sansimonian concept of art as a remarkable spiritual force capable of uniting humanity towards the path of social and civic progress. In those years Liszt also conceived his symphonic music that he called "program music". This was based on the idea that music can never be pure form, flat and expressionless, since it always needs an intense strong ideal that only poetry can provide. Equipping himself with a Dante program, Liszt was thus inspired for his Dante Symphony, composed at Weimar in I856. However, it should not be understood as an homage to the poem, but as a lucid awareness of its modernity on the part of the composer: it is its fragmented and symbolic nature, that significantly excludes the last poem, as well as the ethical value that music should possess, what allows Liszt to select those poetic images that are fit to be infused with an expressive symphonic layer.
\end{abstract}

Keywords: Liszt, program music, sansimonismo, avenirism (Futurism), Symphonic poem. 


\section{LONTANO DALL'ItTAlia E DALla RiVOLUZione: MEDITANDO SU DANTE E SULL' "AVVENIRE»}

lla base della decisione a seguito della quale Liszt acconsentì di trasferirsi
nella città di Weimar come Maestro di corte, ponendosi così alle dipendenze di un Granduca nonostante il suo spirito libero e già fortemente cosmopolita, vi è una ragione squisitamente romantica, che ben si sposa con la biografia del personaggio: un folgorante colpo di fulmine. Liszt infatti, che aveva già da qualche anno rotto con Marie d'Agoult, per colpa delle di lei accuse di infedeltà e libertinismo, nel febbraio del 1847 conobbe a Kiev, in occasione di un concerto di beneficienza, la Principessa Carolyne von SayneWittgenstein, regolarmente sposata con un principe tedesco residente in Russia, madre premurosa di una figlia decenne, Marie. Pretesto dell'incontro fu il ringraziamento che Liszt le volle fare di persona per la cospicua somma di Ioo rubli, da lei donata in occasione del concerto. Egli si recò pertanto nella maestosa dimora di Carolyne, nelle steppe della Podolia, dove ella aveva i suoi immensi possedimenti agricoli, e qui rimase decisamente folgorato dalla sua erudita biblioteca, nella quale non mancavano opere fondamentali di filosofia e di scienza, di religione e letteratura, oltre a testi-chiave nell'immaginario lisztiano come la Divina Commedia e il Faust.

I due si rincontrarono comunque pochi giorni dopo ad Odessa, e si confidarono la loro reciproca fulminea attrazione. All'apice della sua carriera di virtuoso e di solista, solito a calcare i palcoscenici di mezza Europa, Liszt accettò dunque di diventare direttore d'orchestra di un piccolo e alquanto malandato teatrino di provincia, ansioso di costruire un nido d'amore con la prediletta anima gemella. Ma questa non poteva certo essere l'unica motivazione. Il desiderio di trovare una dimora sicura e un approdo quieto dove poter riposare ci restituiscono un'immagine completa dell'uomo-artista, caratterizzato in primo piano da un atteggiamento psicologico di continua inquietudine e irrequietezza, ma allo stesso tempo estremamente assetato di meditazioni, bisognoso di una riflessione spirituale da coltivare anche lavorando con un'orchestra e un coro a sua totale disposizione, approfondendo parallelamente le proprie conoscenze sui materiali musicali non-pianistici, come appunto le compagini sinfoniche, le masse vocali, l'orchestrazione etc.

Senza dubbio il forte sentimento religioso della Principessa Wittgenstein fu una delle qualità che più attrassero Liszt, insieme alla profondità della sua dedizione e al suo pratico vitalismo. Dopo aver di fatto avviato lo scioglimento del suo matrimonio con il nobile tedesco, la Principessa nel giro di pochi mesi lasciò la Russia, in aperta opposizione alle autorità religiose, per unirsi al compositore, non immaginando certo l'isolamento a cui sarebbero stati paradossalmente condannati a causa della loro unione illecita e della convi- 
venza clandestina, spesso snobbata dalla maggior parte degli ambienti sociali da loro frequentati. Un isolamento veramente paradossale trattandosi infatti del principale compositore di corte, nonché fautore di ogni attività artistica della cittadina. I due amanti si stabilirono ad Altenburg, una spaziosa casa non distante dal castello del Granduca; ma la famiglia regnante, che era molto vicina alla Russia -la granduchessa di Weimar, Maria Paulowna, era sorella dello zar- mostrò verso di loro una irriducibile e persistente ostilità tanto da far naufragare l'esperienza ultradecennale di Liszt come Maestro concertatore. Nella lettera al granduca Carl Alexander, l'uomo-artista sfoga tutta la sua amarezza e contrappone la grandezza d'animo di ogni individuo squisitamente romantico alla grettezza degli uomini mediocri, che seguono solo convenzioni di comodo e ragionano esclusivamente in termini di occhiuta convenienza politica:

Il Vostro ambiente, e forse anche la vostra famiglia, Altezza, non trova sicuramente di suo gusto il vedere a Corte un artista che, secondo le parole del principe di Talleyrand è "arrivé et non parvenu", uno che mai si sarebbe potuto comprare con le sue ridicolaggini d'artista i vantaggi d'aver sposato una principessa[...] È chiaro che si vuole impedirmi a qualsiasi prezzo un matrimonio al quale, per la mia nascita, non sono destinato, ma che io credo di essermi guadagnato, e ve lo dico senza falsa modestia. ${ }^{.}$

Il sentimento che univa i due scomodi amanti non necessitava di un riconoscimento giuridico mediante il sacro vincolo del matrimonio, essendo la loro una passione tenuta in vita da un substrato valoriale comune, redenta dall' inesauribile fede in Cristo, e dalla consapevolezza di Liszt di essere avviato lungo un percorso ideale, artistico, personale e di essere votato al progresso dell'umanità e ad un'elevazione spirituale collettiva, anche mediante un rinnovamento radicale del linguaggio musicale tedesco, esperimento tentato e anelato negli anni weimaresi. Ma l'impossibilità di far accettare alla variegata compagine sociale della cittadina tedesca la liceità e ancor di più la necessità di un legame avvertito come assoluto ed indissolubile, amareggiò molto il prolifico Liszt, che in questi anni fu comunque fecondissimo sia nel campo della musica pianistica, che in quello della musica sacra e dei poemi sinfonici. ${ }^{2}$

In realtà non era forse Weimar il luogo ideale per far nascere la nuova musica tedesca, dal momento che per contratto Liszt avrebbe dovuto soltanto dirigere l'orchestra di Corte e prepararne le esecuzioni. Se poi fu anche sovrin-

I. Lettera di Franz Liszt a Carl Alexander, riportata in Dalmonte ( 1983: 68); la lettera è presa da La Mara (1903: $87 \mathrm{sg}$ ).

2. Stupisce come, molti anni dopo, a Liszt non tornò in mente l'amarezza provata in quel periodo, quando la figlia prediletta (e ormai unica rimastagli), Cosima, gli comunicò di voler annullare il matrimonio col marito Hans von Bülow perché attratta da una passione fatale ed inesorabile nei confronti di Richard Wagner; Liszt, che adorava von Bülow come un figlio adottivo, non rivolse a lungo la parola a Cosima e al vecchio amico compositore, considerato come un infingardo rovina famiglie; nei suoi confronti, però, la stima e l'affetto erano sempre stati grandi, come testimonia la dedica a Wagner della Sinfonia Dante. 
tendente artistico delle attività musicali della città lo si deve alla sua tenacia avvenirista nella ricerca della nuova musica; non va però dimenticato che il principio saint-simonista della funzione educativa dell'arte, appreso da Liszt negli anni parigini, era destinato a scontrarsi con un organico assai carente, quando non inadatto ai traguardi che il nuovo direttore artistico sognava di raggiungere. E una politica culturale avvenirista e idealmente proiettata nel futuro non aveva certo vita facile in una dimensione politico-istituzionale estremamente rigida e conservativa, lontana dalle cannonate rivoluzionarie che da lì ad un anno avrebbero squassato quegli ordinamenti restaurati dopo le guerre napoleoniche e considerati vetusti ed iniqui. Chiusa in un'autoreferenzialità culturale ed estetica, dedita soltanto a badare al mantenimento di privilegi secolari e al prestigio della casata regnante, Weimar era una cittadina immobile e sonnecchiante, impermeabile alle idee illuminate della Rivoluzione che pure in un primo momento, all'inizio del secolo, avevano fatto interrogare le classi dominanti dell'aristocrazia tedesca sull'opportunità di importarne le più innocue conquiste sociali, per svecchiare, anche in chiave nazionalistica, il volto della Germania, regalandole un assetto nuovo; la reazione, invece, vinse in tutta Europa, e a maggior ragione a Weimar, "proprio appoggiandosi alla paura dell'alta borghesia di perdere i suoi modesti privilegi e alla stanchezza della gente stremata da rivoluzioni, sommosse e imprese militaresche». ${ }^{3}$

Tuttavia Liszt elesse questo centro così appartato e chiuso come luogo di promozione dell'arte, e cercò di utilizzare l'istituzione che era chiamato a presiedere come il motore di uno sviluppo culturale ed estetico, secondo il principio per cui quanto più si trionfa su un pubblico dagli orizzonti di gusto limitati e conservativi, tanto più facile sarà diffondere il nuovo linguaggio presso gli altri: in questa decisione giocò appunto un ruolo fondamentale l'influsso delle teorie sansimoniste, conosciute durante gli anni della formazione parigina. Pur non partecipando mai con atti concreti ai moti rivoluzionari, la cui scintilla scoccò nel luglio del 1830 proprio nella capitale francese, Liszt aderì convintamente a quello spirito umanitario che inquadrava il progresso dell'arte come destino collettivo della storia umana, nel suo divenire; avvicinando la propria concezione dell'arte a quella sansimoniana della scienza, quale forza capace di unire gli uomini nel cammino comune del progresso, Liszt individuava il duplice scopo della musica: da un lato elevare e consolare l'uomo, dall'altro benedire e glorificare Dio. Per arrivare a questo scopo era pertanto:

[...] imminente la creazione di una musica nuova, essenzialmente religiosa, forte ed efficace. Questa musica che, in mancanza d'un altro termine, chiameremo umanitaria riassumerà in proporzioni colossali il TEATRO e la CHIESA; sarà allo stesso tempo drammatica e sacra, pomposa e semplice, patetica e grave, ardente e sfrenata, tempestosa e calma, serena e tenera.[...]

3. Dalmonte (1983: 56). 
Sì, non ne dubitiamo, ben presto sentiremo esplodere nei campi, nei casolari, nei villaggi, nei quartieri, nelle officine e nelle città, canti, cantici, arie, degli inni nazionali, morali, politici, religiosi, fatti per il popolo, insegnati al popolo, cantati dai lavoratori, dagli artigiani, dagli operai, dai ragazzi e dalle ragazze, dagli uomini e dalle donne del popolo. Tutti i grandi artisti, poeti e musicisti daranno il loro contributo a questo repertorio popolare che si rinnoverà incessantemente.[...] Sarà il FIAT LUX dell'arte ${ }^{4}$.

In un connubio di neocattolicesimo francese e prime teorie di stampo socialista-umanitario, Liszt promuoveva un'arte sociale che indicasse all'umanità una strada, e che ne accompagnasse il cammino mediante suggestive epopee simboliche: il pellegrinaggio, l'itinerario spirituale, la contemplazione estatica, la musica a programma avrebbero dovuto sempre assolvere questo duplice compito, religioso e sociale.

La preoccupazione dell'artista è sempre rivolta alle masse, e la musica a programma deve essere pensata per comunicare pensieri, sentimenti e contenuti vòlti al progresso civile ed umano: l'elemento poetico diventa il riferimento principe nella creazione della nuova musica lisztiana, perché solo congiunte musica e poesia possono incarnare lo spirito dei tempi, abbracciare «religiosamente tutto il corpo sociale» e prendere pienamente possesso «di quell'ampia eredità loro provvidenzialmente assegnata»s. In un'epoca storica in cui la civiltà strumentale esplorava le sue potenzialità e il pensiero estetico romantico predicava la superiorità assoluta della musica nel campo delle arti, l'esigenza di unire poesia e suoni, in un contesto puramente sinfonico (senza ricorrere quindi al melodramma) non deve essere intesa come una carenza del mezzo espressivo musicale, che non basta da sé, non è più autosufficiente; è invece l'idea che la musica non potrà mai essere pura forma, piatta ed inespressiva, ma avrà sempre bisogno di un contenuto ideale forte, che solo la poesia potrà darle; e questo contenuto sarà funzionale alla forma, nel senso che ne garantirà l'ispirazione e la creazione. ${ }^{6} \mathrm{Nel}$ famoso scritto sulla musica a programma di Berlioz, Liszt chiarisce questo punto del suo pensiero:

Il programma non ha altra funzione se non quella di indicare a scopo preparatorio i momenti spirituali che hanno spinto il compositore alla creazione della sua opera e i pensieri ai quali ha cercato di dare corpo attraverso il programma[..] Il sinfonista poetante che si pone il compito di rendere altrettanto chiara e distinta quanto lo è nel suo spirito, un'immagine, una successione di stati d'animo di cui è inequivocabilmente e fermamente cosciente, perché non dovrebbe tendere alla completa comprensione con l'aiuto di un programma? ${ }^{7}$

4. Franz Liszt, De la situation des artiste et de leur condition dans la société, in Liszt, (1987: 66-67).

5. Franz Liszt, Encore quelques mots sur la subalternité des musiciens, in Liszt (1987: 85-86).

6. A proposito della centralità della musica a programma nel pensiero estetico lisztiano fondamentale è il capitolo Liszt's Prayer contenuto nel libro di Berthold Hoeckner (2002: 155-188).

7. Franz Liszt, Berlioz und seine "Harold Symphonie", in Liszt, (1987: 359); il saggio fu pubblicato 
L'ausilio di un programma, ossia di una suggestione, di un'immagine, di un sogno utopistico che l'artista sente nascere dentro di sé, è perciò una parte costitutiva del nuovo linguaggio musicale che Liszt si propone di creare, poiché senza di esso l'opera d'arte in musica perderebbe il suo potenziale eversivo, l'impegno etico, civile e religioso, e infine la sua missione educativa. Da questo punto di vista, la scelta di un'epopea spirituale come quella che compie Dante nelle tre cantiche della Commedia non sembra condizionata dal valore letterario e simbolico dell'opera stessa, che in piena temperie romantica era letta specialmente in chiave nazionalistica, quanto invece dalle potenzialità espressive che quell'itinerario dell'anima, quel viaggio umanissimo ma allo stesso tempo ultramondano, potevano suggerire al compositore, in quegli anni weimaresi così deludenti e frustranti, che però furono anche culla dei suoi sogni avveniristici.

\section{Simbologia E Frammentarietà:}

\section{DANTE NEL PENSIERO LISZTIANO}

Gli anni giovanili "di pellegrinaggio" in Italia contribuirono notevolmente alla creazione di una visione fortemente idealizzata e sublimizzata della patria delle lettere e della musica cristiana: lo stupore di fronte alle meraviglie naturali, per il patrimonio storico-artistico e per l'infinita bellezza dei paesaggi italiani, sospesi tra arte e natura, suggerì a Liszt una serie di riflessioni, alcune delle quali sembrano perfette cartoline del Belpaese:

Se c'è un luogo al mondo in cui il rumore esteriore delle cose non penetra affatto, se c'è un posto isolato che le dispute vane e le ambizioni puerili non potrebbero turbare, questo è, sicuramente, il luogo da cui scrivo, è il posto isolato in cui mi sono fermato per dare un ultimo addio all'Italia, per gioire un'ultima volta dell'ineffabile bellezza di questa terra amata dalla luce. ${ }^{8}$

Fu probabilmente nei giardini all'inglese di Villa Melzi, a Bellagio, che Liszt respirò per la prima volta il profumo della poesia di Dante, leggendone passi all'ombra dei platani, o ammirando la scultura di Bonelli raffigurante Beatrice che indica il cielo tenendo per mano lo sperduto viaggiatore ultraterreno. $\mathrm{Ma}$ a Bellagio Dante doveva ancora corrispondere alla figura mitica tratteggiata dal romanticismo francese: come ha scritto Rossana Dalmonte, «doveva essere più il Dante della Vita nova che non della Commedia».9 e doveva essere sicuramente già passato nello specchio deformante degli scritti e delle immagini di Victor Hugo, Balzac, M.me de Staël, nonché della prediletta compagna di Liszt

a puntate sulla rivista Neue Zeitschrift für Musik nel 1855 .

8. Così scriveva Liszt all'amico Hector Berlioz il 2 ottobre 1839, da San Rossore, dove si era fermato durante il viaggio di ritorno; cfr. Liszt, (1987: 218).

9. Cfr. Dalmonte (20I2: 56 ). 
d'allora, Marie d'Agoult. Era quindi Dante nella sua dimensione eroica di esiliato, di mistico incompreso, cui si guardava con attenzione per il suo rapporto con Beatrice, un amore purificante, metafisico. Probabilmente tale approccio alla figura del poeta fiorentino mutò in seguito al primo soggiorno romano. ${ }^{10}$

Quando parliamo della ricezione lisztiana di Dante ci muoviamo purtroppo sempre nel campo delle ipotesi: contrariamente ai numerosi scritti saggistici e critico-musicali, non ne disponiamo, o forse non è mai esistito, nessuno in cui appaia la sua posizione nei confronti del poeta fiorentino. Dobbiamo perciò concentrarci sul suo pensiero musicale, e ovviamente sul linguaggio compositivo presente nelle opere dedicate a Dante.

Nell'opera di Liszt dedicata a Dante appaiono due aspetti significativi, uno di carattere formale e l'altro nominalistico. Da un lato è interessante notare come il primo titolo di quella che diventerà la Dante-Sonata - vale a dire l'ultima composizione del secondo volume delle Années de pèlegrinage (Après une lecture du Dante)- fosse semplicemente Fragment, ossia un brano libero, quasi fantastico, cui è impossibile dare una forma unitaria né ricondurre a un insieme, che resta, per l'appunto, un frammento; dall'altro abbiamo invece l'interessante progetto, mai realizzato, di un grande Dramma lirico basato sulla Commedia, da portare sulle scene weimaresi con libretto di Autran e i disegni di Bonaventura Genelli ${ }^{\text {II }}$. Alla base del progetto vi era l'idea di rappresentare sulla scena teatrale in forma di dramma il viaggio nei tre regni dell'aldilà, con l'utilizzo dei necessari pezzi chiusi di musica vocale (arie, duetti, cori), alternati ad episodi puramente strumentali; ma tale lavoro sarebbe risultato troppo lineare e logico, con una drammaturgia rigidamente plastica, presente anche fisicamente nel diorama che avrebbe dovuto dare forma tridimensionale agli schizzi di Genelli. Ciò che Liszt voleva sottolineare era invece un altro aspetto: la modernità del poema dantesco, ossia la sua capacità di «non corrispondere ad alcuna definizione», ${ }^{\mathrm{I}}$ e quindi, ancora una volta, la sua frammentarietà, la sua natura pittorica, episodica, e non monumentale. A Liszt non interessava la costruzione della Commedia nel suo insieme, cioè la salita dalle selve infernali all'Empireo, e poi su fino al cospetto di Dio, bensì alcuni episodi specifici,

IO. Molto significativo, da questo punto di vista, è il suddetto studio critico di Rossana Dalmonte, che pone in evidenza l'influsso esercitato sul pensiero lisztiano da parte della cosiddetta scuola pittorica dei Nazareni e da alcuni artisti francesi frequentati a Villa Medici, come il pittore Ingres, che realizzò dei ritratti di Liszt e di Marie; un tratto stilistico estremamente delicato quello dei Nazareni, quasi preraffaelita, che non solo riportava la pittura ad uno stadio primitivo, ma voleva idealmente collegare la loro arte agli albori del cristianesimo, senza mediazioni di maniera, al fine di far emergere nettamente le dicotomie e i contrasti.

II. Pittore ed incisore nato a Berlino, formatosi a Roma e al servizio presso la corte di Weimar, dove morì nel I868; notevoli furono i suoi disegni, per lo più a semplice contorno, e i suoi acquerelli, molti dei quali ispirati ad episodi della Commedia e dei due poemi omerici.

I2. Cfr. Dalmonte (1983: 67). 
grazie al valore simbolico, etico, religioso dei quali potesse esprimere musicalmente l'eterna dicotomia tra bene e male, senza doversi attenere rigidamente alla struttura e allo svolgimento lineare delle tre cantiche. La ricerca e la scoperta di Dante, come percorso incessante e continuo, che accompagnò Liszt durante l'intera esistenza, rappresentano forse al meglio quell'ideale filo rosso tra musica, poesia e pittura che il pianista-compositore considerava elemento base della musica dell'avvenire, poiché solo grazie alla sinergia di questi tre elementi l'ascoltatore avrebbe potuto comprendere al meglio le immagini e gli stati d'animo che in quella musica erano depositati. Accogliere la simbologia ultramondana del poema dantesco significava pertanto dotare il potente mezzo espressivo sinfonico della forza prorompente della poesia e delle immagini da essa suscitate. Per ottenere questo, non era poi così importante la struttura cronologica del poema, quanto la sua natura episodica, non tanto la sua ascensionalità dall'oscurità alla luce, quanto l'esemplarità e la forza simbolica di alcuni frammenti; per questa ragione la conclusione della Dante Symphony non è, come logicamente ci aspetteremmo, nel Paradiso. ${ }^{13}$

La critica novecentesca ha da tempo risolto il problema dell'unità della Commedia individuandone il senso allegorico complessivo come annuncio etico-politico ad un'umanità in crisi, bisognosa di ritrovare un ordine; tale ordine sarebbe stato possibile solo nel momento in cui la Curia romana avesse rinunciato alle ambizioni temporali, spettanti soltanto all'Impero. Le diverse pagine dell'itinerario oltremondano, che conoscono momenti differenti di intensità espressiva, non ammettono una lettura frammentaria del poema: gli episodi più suggestivi non sarebbero ciò che sono se non fossero inseriti nella cornice completa dell'opera. A riprova dell'unitarietà del poema basti pensare alla suddivisione dell'aldilà in tre regni distinti, cui corrispondono il disperante dolore, l'attesa della salvezza, la piena letizia della comunanza con Dio; oppure alla partizione in 33 canti, cui si aggiunge un proemio generale per raggiungere il numero perfetto di Ioo, secondo la numerologia medievale; o ancora all'argomento politico presente nel sesto canto di ogni cantica, e alla parola stelle con cui ogni cantica si conclude. Infine, la natura allegoricodidattica del poema è determinata da un forte realismo: la metafora dell'oltremondo, come ha scritto Giorgio Inglese, è specchiata nel nostro mondo, per cui esso è reso sensibile e leggibile con forme e modi realistici; le anime che si presentano sulla scena come exempla di vizi e virtù «esibiscono fisionomia e passioni di viventi». ${ }^{14}$ Dante ha perciò creato personaggi reali, caratterizzati da situazioni psicologiche proprie della vita terrena, quindi riconoscibili pur attraverso la trasfigurazione oltremondana data dalla dimensione dell'aldilà.

13. A tal proposito rimando all'ottimo saggio di Antonio Rostagno, in cui non manca una piccola ma significativa parte relativa al poema lisztiano; si veda Rostagno (2013: 175-24I).

I4. Inglese (2007: I0). 
È questo aspetto, così umano e terreno, valido in ogni epoca storica, il vero programma poetico da trasporre in musica che attrasse Liszt.

\section{ANAlisi dell'opera}

L'iniziale progetto "multimediale" di suoni ed immagini, che balenò nella mente di Liszt subito dopo aver visto la serie di schizzi ispirati a Bonaventura Genelli dal poema dantesco, e che prevedeva la proiezione tridimensionale dei luoghi, delle persone e degli oggetti che la musica avrebbe illustrato sonoramente, grazie all'ausilio della recente invenzione del diorama, fu abbandonato per la perenne carenza di fondi della corte di Weimar; invece l'idea di una "Sinfonia Dante" sopravvisse e venne realizzata nel I856, dopo la grandiosa Faust Symphony del 1854. La prima esecuzione ebbe luogo a Dresda l'anno successivo, nel 1857, sotto la direzione dello stesso compositore. La sinfonia, ispirata dalla Commedia, si divide in due parti: una prima sezione, denominata Inferno, e una seconda composta dal Purgatorio e da un coro finale "Magnificat" per voci bianche o sole voci femminili. Il coro sostituisce il Paradiso, che essendo regno assoluto della divinità non può essere rappresentato neanche dalla musica. L'idea di concludere il lavoro sinfonico con un episodio corale di chiara ascendenza beethoveniana parve non convincere molto Wagner, cui l'opera è dedicata. Sappiamo infatti che per lui il movimento finale con soli e coro della Nona sinfonia beethoveniana ne costituiva la parte più debole, e d'altra parte l'alto contenuto teologico e simbolico che il regno dell'Empireo portava con sé rappresentava un'impresa smisurata anche per Dante stesso, per cui l'ultima cantica sarebbe la meno riuscita. ${ }^{\text {Is }}$

Ma indipendentemente dal consiglio dell'amico, Liszt non avrebbe mai potuto, per la sua forte dimensione religiosa, delegare ad un movimento di sinfonia la visione delle schiere angeliche e della rosa dei beati, poiché certi misteri restano intellegibili, ed è naturale che sia così; ma la lode a Dio non può essere estromessa, e per questa ragione l'opera si conclude con un $\mathrm{Ma}$ gnificat, aspirazione delle anime purganti e penitenti che ancora attendono un giudizio definitivo e sperano sino all'ultimo nell'infinita clemenza divina.

Prima di addentrarci in una pur sommaria analisi dell'opera, occorrerà chiarire, un'ultima volta, il pensiero di Liszt a proposito della finalità $\mathrm{e}$ dell'estetica della musica a programma, ben delineato, come abbiamo visto,

I5. Wagner doveva ben conoscere il famoso verso dantesco, nell'ultimo canto del Paradiso, quando l'invenzione poetica e la fantasia pittrice dell'autore si arrendono esplicitamente di fronte alla grandezza del mistero divino: "A l'alta fantasia qui mancò possa», quartultimo endecasillabo del poema, sta ad indicare, infatti, come alla facoltà dell'immaginazione, capace di mediare tra ciò che gli occhi di un uomo vedono e ciò che il suo intelletto riesce a comprendere, viene meno alfine l'aiuto divino, e le risulta impossibile riportare in un linguaggio terreno un mistero che va al di là della ragione umana. 
nell'amplio saggio sull' Harold en Italie di Berlioz: è importante ricordare come l'assunzione di una idea poetica centrale, o di un soggetto significativo che si vuole descrivere e assumere come riferimento, venga considerata un mezzo e uno strumento da adoperare per scardinare i vincoli formali dell'opera sinfonica tradizionale.

La ripresa, l'alternanza o la variazione di temi melodici non sono perciò dettate da esigenze di architettura compositiva, bensì dall'incessante condizionamento dell'idea poetica, che impedirà all'ascoltatore di cadere in interpretazioni errate rispetto all'intento originario del compositore; se pertanto lo spunto extramusicale può essere identificato dal titolo della sinfonia, o da alcuni versi poetici disseminati nella partitura, esso non potrà determinare l'andamento della musica, ma sarà casomai la musica a scorrervi parallelamente, al fine di valorizzare l'idea poetica servendosi di risorse espressive, ritmiche e dinamiche.

Lungi dal voler trasferire in musica un capolavoro della letteratura, cercando di tradurre nel linguaggio dei suoni i suoi caratteri linguistico-semantici, Liszt ha voluto proseguire, nella Dante Symphony, il percorso già intrapreso con i cicli pianistici, verso una lingua musicale narrativa e pittrice, servendosi stavolta dell'organico orchestrale e di una tavolozza di colori che il pianoforte da solo non poteva dargli. Il passaggio dal microscopico al macroscopico non fu certo indolore per il nostro compositore, tuttavia il cammino non poteva che farsi più irto e avvincente, dilatando i parametri agogici, dinamici e timbrici su di una scala immensamente maggiore e finora inesplorata.

Come avremo modo di vedere, la scrittura orchestrale di questo capolavoro lisztiano è costruita intorno ad alcune isolate e specifiche immagini poetiche, su cui insiste una dimensione agogica, fatta di respiri e fluidità del tempo, ed una dimensione timbrico-dinamica: l'orchestra raggiunge infatti una piena sonorità nelle pagine dei "tutti", ma conosce anche momenti di affettato lirismo e di piglio più cameristico, isolando sezioni strumentali accomunate da un timbro e da un colore assai simili. Se quindi i soggetti letterari sono noti, e il programma non ha altro scopo se non quello di indicare all'ascoltatore determinati pensieri ed immagini che solo con la musica possono rivelarsi, a fare un passo in avanti in questa operazione poetico-musicale non è tanto l'elemento extramusicale, quanto proprio la musica, per il cui progresso è indispensabile il programma: non stupisce, quindi, come la terza cantica dantesca venga tagliata fuori, perché non così necessaria ai fini della missione avvenirista, quanto invece può esserlo il testo del Magnificat con l'intervento della compagine corale (senza però sfociare nel melodramma); tale sezione, che rimane una suggestione e uno stato d'animo a sé, tuttavia non sarebbe comprensibile se non fosse posta come traguardo del cammino psicologico e spirituale che l'opera tutta ha percorso. Sarà necessario, dunque, tenere sempre 
ben presente come l'uso di alcuni effetti timbrici, dei raddoppi, dei registri estremi, dei cromatismi, delle dissonanze, di organici inusuali e di sonorità evocanti immagini e sensazioni suggestive, sarà un uso sempre funzionale alla musica stessa, al suono di per sé: saranno i temi e le idee melodiche a far parlare il programma, non esso a dar voce alla musica; e l'itinerario psicologico e spirituale (ma anche drammatico) che è indicato dal titolo, dal programma, dai riferimenti testuali presenti nel brano, sarà delineato dalla musica stessa, nel suo permutare ritmico, melodico ed armonico.

Tale itinerario muove i suoi passi sin dal primo movimento, l'Inferno, affidando all'orchestra un commento orchestrale alle parole oscure scolpite sulla porta d'entrata al regno avernale, le stesse che costituiscono l'incipit del terzo canto. Si comprende facilmente il perché di questa scelta e della conseguente omissione sia dello smarrimento nella selva oscura, sia dell'incontro con Virgilio, sua guida spirituale: a Liszt interessava la dimensione frammentaria ed episodica del poema dantesco, come già abbiamo visto, e quindi l'aspetto metrico del testo dell'iscrizione e l'arcano significato delle parole acquistano il massimo rilievo.

L'assetto metrico garantisce un effetto di tensione, costituito dall'esordio improvviso della prima terzina del canto, e quindi un'iterazione anaforica di un primo quinario tronco e di un secondo emistichio, un settenario piano, che introduce un'atmosfera disperante, cupa ed emotivamente significante all'intero episodio ("Per me si va / nela città dolente; per me si va / nel'etterno dolore" etc. $)^{16}$; ma è l'intero sistema metrico dantesco, ossia la famosa terzina di endecasillabi a rima incrociata, che Liszt assume come riferimento linguistico da rivestire sonoramente. La sinfonia si apre infatti con un cupo disegno melodico, dal timbro scuro e arcano, in cui già appare la cellula musicale della terzina di crome, che si presenterà molto spesso nel corso del primo movimento in differenti tipologie mensurali: questo canto sillabico, incalzante ma sofferente, accompagna fedelmente il verso dantesco, riportato nella partitura, secondo la migliore tradizione della musica a programma, ed è affidato a strumenti gravi e dal colore intenso e corposo come tromboni tenori, trombone basso, tuba, oltre che agli archi scuri, alle viole, ai violoncelli e ai contrabbassi.

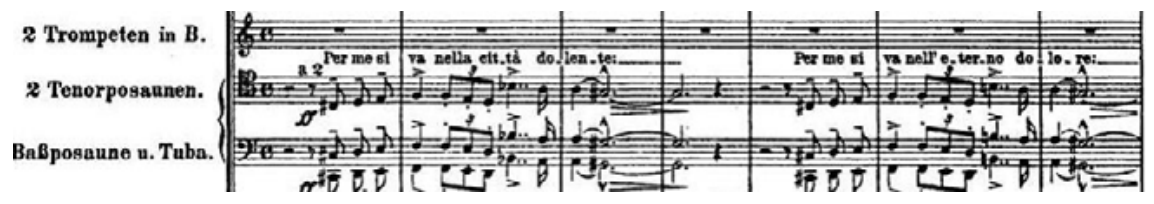

I6. Per il testo del poema dantesco faccio riferimento alla recente edizione critica dell'Inferno, curata da Giorgio Inglese; cfr. Inglese (2007). 
È da notare come l'espressione sonora che Liszt vuole conferire a queste terzine dantesche, da sempre connotanti il regno infernale come luogo di non-ritorno nell'immaginario collettivo, si avvalga di una melodia sillabica e dell'intervallo musicalmente più semplice, ossia l'unisono. Ecco infatti la parte degli archi scuri:

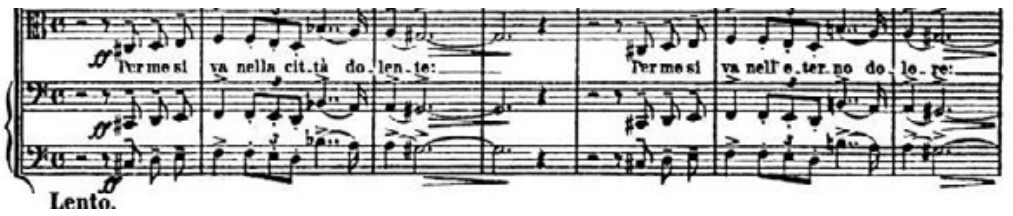

La musica continua a rivestire il testo, incalzando ritmicamente e dinamicamente l'intero corpo orchestrale che infine esploderà con potentissimi accordi sulle parole finali dell'iscrizione avernale: "Lasciate ogni speranza, voi ch'entrate". Il madrigalismo lisztiano procede con delle puntute scale cromatiche eseguite dagli archi scuri che, per mezzo di continui disegni di tensione e allentamento, porteranno infine all'emergere di un colossale accordo dissonante a piena orchestra:

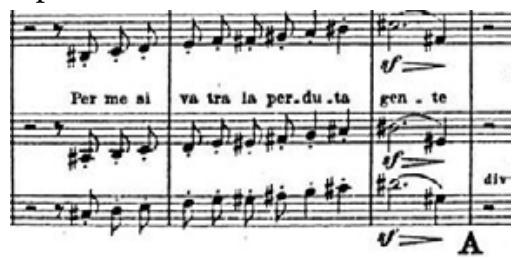

L'ingresso nella dimensione ultraterrena è scandito da un progressivo accelerando del disegno melodico, che procede per progressioni cromatiche e nervosi ostinati di terzine affidati ai bassi; il programma originario di musica illustrativa di un dramma lirico, o comunque di una proiezione mediante diorama di figure ed immagini esplicative, risulta ancora ben presente nella partitura, che riveste il testo poetico per evocare sonorità e timbriche orchestrali vòlte principalmente alla semantica del «programma»; le progressioni sono infatti sempre discendenti, frananti, e le armonie incerte e cedevoli si nascondono dietro ad ostinate figurazioni terzinate, a ricordarci sempre qual è la matrice metrica su cui il testo è fondato. La sensazione di un castigo eterno, di una caduta rovinosa e irreversibile perché in vita non si è voluto rinunciare ai peccati più seducenti, emerge nettamente in una dinamica e in un'agogica tempestosa, come il destino che attende i dannati

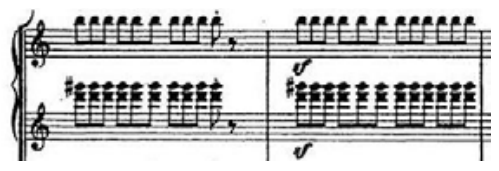




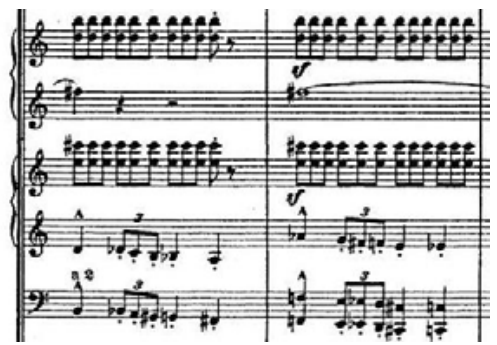

Ma secondo la migliore tradizione dantesca (e anche in un'ottica sinfonica di blocchi contrastanti), la legge del contrappasso può dar vita a rovesciamenti insoliti: così, da una scrittura sinfonica compatta, fondata su masse sonore e blocchi timbrici ben delineati e sempre nell'idea di una musica pittorica capace di illustrare il testo fondante, si passa ad un altro frammento del poema dantesco, il canto di Paolo e Francesca; il loro amore idealizzato e mai consumato necessita di un altro colore sinfonico, di una deliziosa intimità e di maggiore dolcezza, e non può più servirsi di sonorità ostentatamente gravi, marziali, quasi sproporzionate.

Se perciò il tumultuoso movimento veloce si avvia al suo culmine, procedendo nel suo ostinato disegno di terzine,

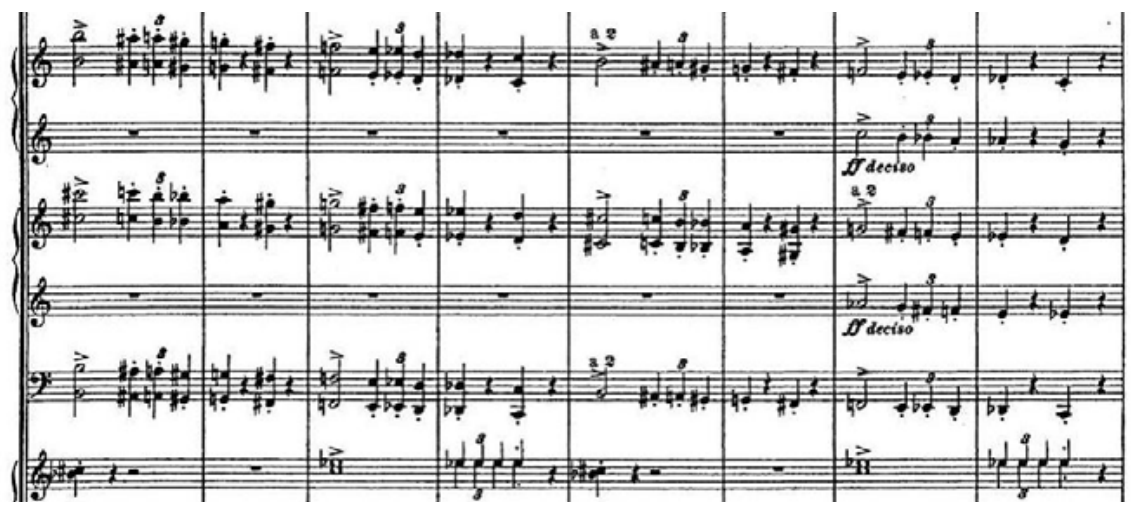

ecco che una linea melodica morbida ed avvolgente accompagna le parole degli incauti amanti, ${ }^{17}$ affidata al suono malinconico di un corno inglese, e sorretta dai dolci arpeggi pizzicati dell'arpa:

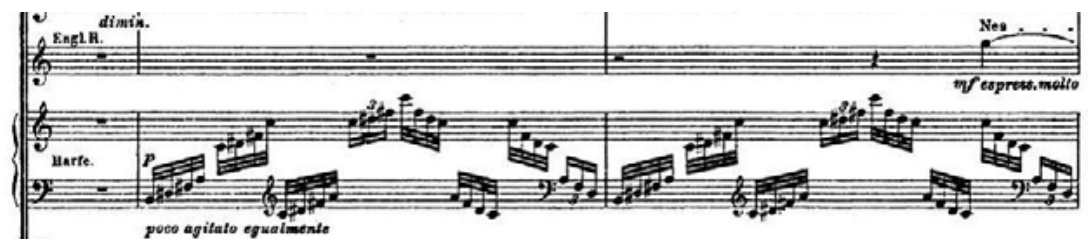

17. V canto: «Nessun maggior dolore/che ricordarsi del tempo felice/nella miseria ». 


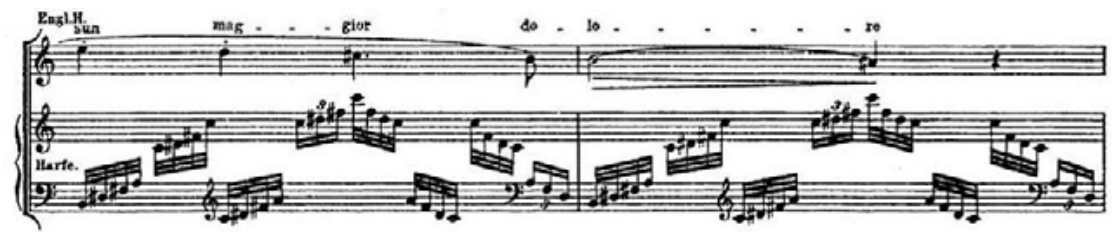

Il rimpianto del tempo felice è legato ad una dimensione contingente, terrena, mentre ad essere eterna è la miseria: per questa ragione la melodia risulta ugualmente dolente, pur nella sua apparente dolcezza.

La speranza di una redenzione ultima, e di un clemente perdono finale, pervade invece il secondo movimento della sinfonia, il Purgatorio, ossia quel regno ultramondano dove lo spirito umano, pur indegno di accedere al Paradiso, si purifica e si prepara degnamente ad una futura ascensione: qui il clima appare sospeso, ma gli elementi idiomatici del ritmo terzinato compaiono ancora, sempre affidati all'arpa, mentre prima l'oboe e in seguito il corno inglese intonano una frase melodica di ampio respiro.

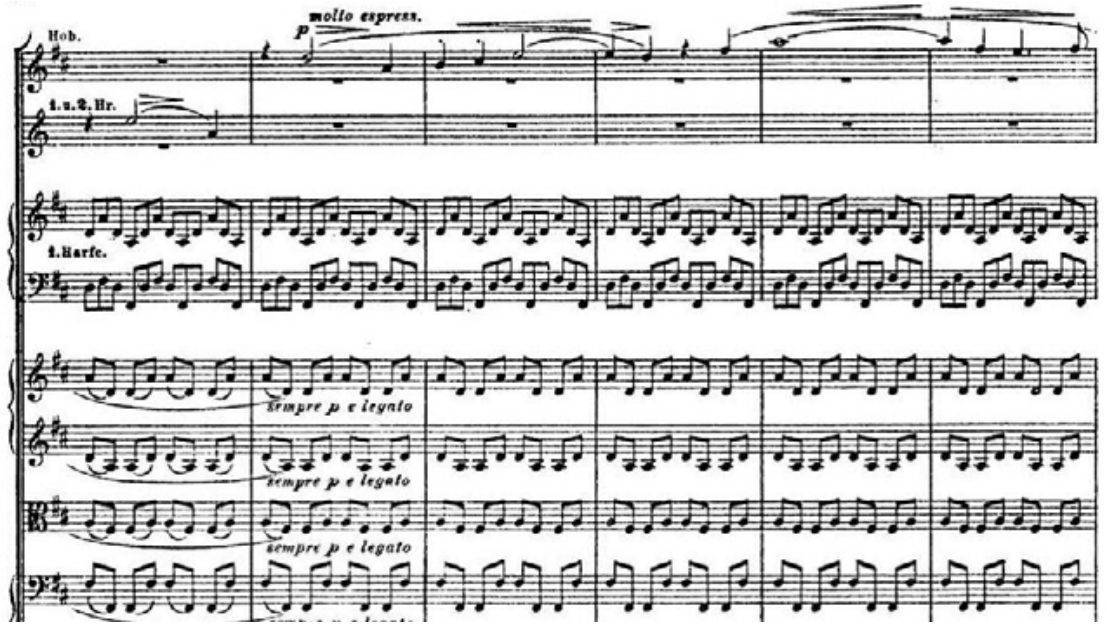

Il contrasto tra le terzine dell'arpa e le duine di crome degli archi è una costante anche nel coro finale della sinfonia, come un disegno incessante che non ha culmine e che promette un approdo, in quanto attesa di felicità e mistero senza soluzione. Come la sua fede, come il suo progetto di musica dell'avvenire, come la sua incessante ricerca di equilibrio tra arte e religione, così la sua sinfonia si arrende di fronte al mistero divino, e si limita soltanto a celebrarne la grandiosità, senza illudersi di comprenderla.

Il Lamentoso che conclude la sezione dedicata al secondo regno dantesco è scritto come un fugato inquieto e instabile, come il destino incerto delle anime purganti. Infine il messaggio poetico prende forma attraverso un testo che è intonato da un coro di voci femminili (o voci bianche), ma il "program- 
ma» è qui extradantesco, perché non c'è musica che possa esprimere la visione dell'Empireo. Liszt sceglie perciò di affidarsi ad una classica preghiera di invocazione e lode, con testo latino, che non certo casualmente è una cantica, come le tre sezioni del poema dantesco; e se non è possibile illustrare con la musica il regno di Dio, il Magnificat può degnamente riassumere l'itinerario spirituale dell'anima che questa sinfonia vuole suggerire, concludendo idealmente la triade dantesca.

Il contrasto tra le duine e il disegno terzinato accompagna costantemente l'intonazione della preghiera di lode, significativamente affidata ad un timbro vocale chiaro e melodioso. Se la numerologia medievale di stampo cristiano assume qui un valore simbolico non è dato saperlo. Il dubbio tuttavia permane.

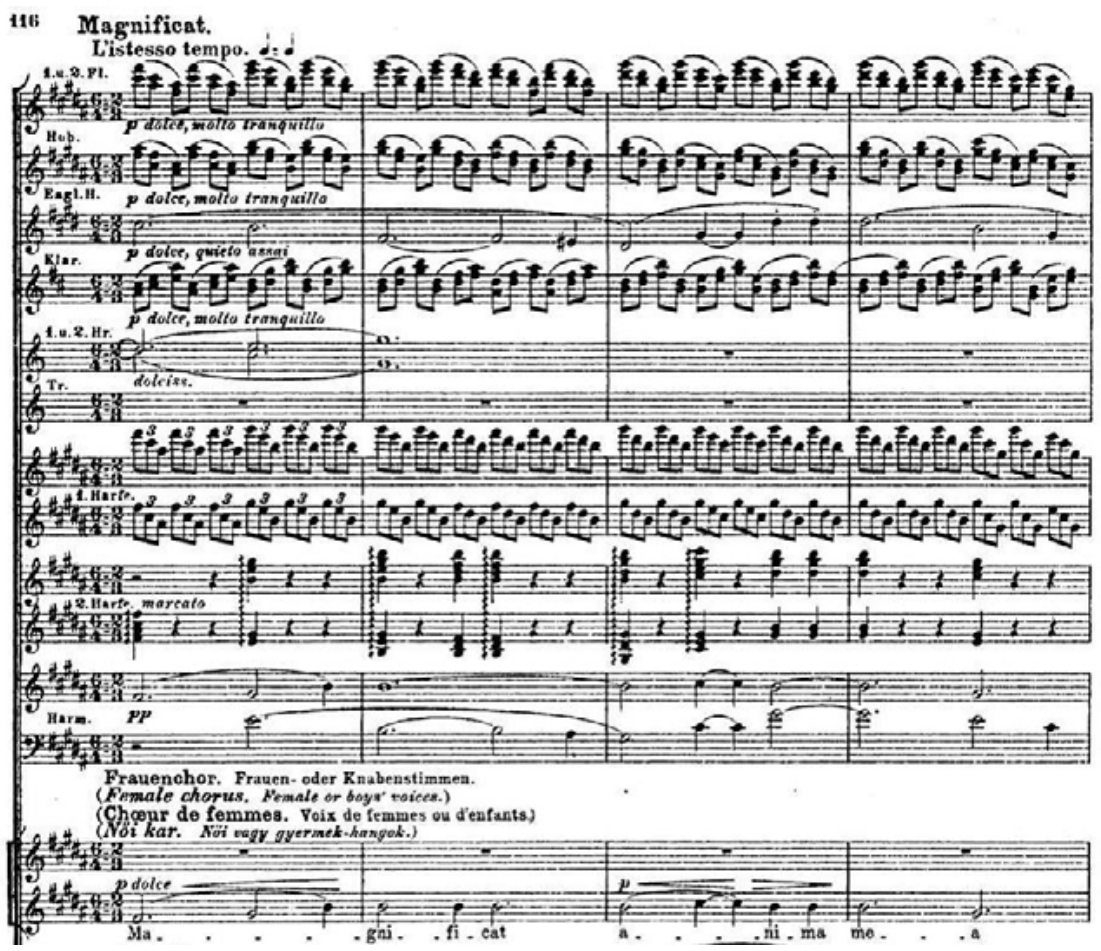

Alla fine di questa sommaria analisi di questa pur preziosissima opera sinfonica a programma di Franz Liszt, restano dunque poche certezze: circa il rapporto del compositore con Dante e la sua poesia molto è stato scritto ma ancora tanto è da scoprire; lo stesso vale per il rapporto tra il testo letterario o poetico come fonte di ispirazione compositiva e la sua traduzione in musica nei poemi sinfonici e nelle sinfonie a programma. La ricerca costante, nell'ambito di una fede religiosa che così fortemente caratterizzò l'uomo e l'artista Franz 
Liszt, di un nuovo linguaggio musicale e di nuove potenzialità espressive delle forme compositive resta senza dubbio l'aspetto più affascinante di quest'opera e di molte altre produzioni sinfoniche "a programma", che forse sono state troppo a lungo dimenticate e meriterebbero indagini più approfondite, quali unici documenti di un pensiero musicale lisztiano che altrimenti rischiamo di perdere.

\section{BiBLIOGRAFIA}

Inglese, G., 2007 = Dante Alighieri, Commedia. Inferno, a cura di Giorgio Inglese, Carocci, Roma, 2007.

Dalmonte, R., 1983, Franz Liszt. La vita, l'opera, i testi musicati, Milano, Feltrinelli.

—, 20I2, Franz Liszt alla ricerca di Dante, in Nel cosmo di Franz Liszt. Raccolta di scritti in onore di un musicista dell'avvenire, a cura di Claudia Colombati e Maurizio d'Alessandro, Roma, Aracne.

Hoeckner, B., 2002, Programming the Absolute, Princeton-Oxford, Princeton University Press, 2002.

La Mara, (a cura di), 1903, Briefwechsel zwischen Franz Liszt und Carl Alexander Grossherzog von Sachsen, Lipsia, Breitkopf u. Härtel.

Liszt, F., 1987, Un continuo progresso. Scritti sulla musica, Milano, Unicopli.

Rostagno, A., 2013, Dante nella musica dell'Ottocento, in Atti e memorie dell'Arcadia, Roma, Edizioni di Storia e Letteratura, 2, pp. 175-24I. 